\title{
Fitness-Related Resistance Training-with which Training Intensities do Recreational Athletes Train?
}

\author{
Christoph Eifler* \\ Department of Applied Training Science, German University of Applied Sciences for Prevention and Health Management (DHfPG), Germany
}

Received: April 09, 2018; Published: April 19, 2018

*Corresponding author: Christoph Eifler, Department of Applied Training Science (Fachbereich Trainingswissenschaft),German University of Applied Sciences for Prevention and Health Management (Deutsche Hochschule für Prävention und Gesundheitsmanagement-DHfPG),Hermann Neuberger Sportschule 3,Germany

\section{Abstract}

Training intensity is considered to be one of the most important loading parameters in strength training.This also applies to fitness-related strength training in commercial fitness clubs. More than 10 million recreational athletes perform fitness-related resistance training in German fitness clubs. However, hardly any evidence can be found on the implementation of scientific training recommendations to control the training intensity of resistance training within this setting. The common used method to control training intensity in fitness-related strength training is to quantify the athletes' rate of perceived exertion (RPE). It is widely unknown if this approach to control training intensity is in accordance to the recognized recommendations in the relevant scientific training literature. This gap in research was a desideratum for a multicenter-study in German fitness clubs.

One branch of research in this study was the evaluation of the realized weight loads of recreational athletes working with RPE-based training intensity in relation to the one repetition maximum (1-RM) as a recognized reference value to control training intensity in strength training. Training intensitiesof 211 recreational athletes ( $\mathrm{N}=99$ women, $\mathrm{N}=112 \mathrm{men}$ ) were analyzed. The average realized training intensity over a training period of six weeks was $54.50 \%$ of $1-\mathrm{RM}( \pm 6.58)$. This training intensity lies far off from any scientific training recommendations and is clearly too low. It must be called into question, if strength training with such low intensity is an effective and efficient workout to reach the expected training goals of fitness customers. These study results confirm the need for evidence-based strength training protocols in fitnessrelated resistance training.

Keywords: Fitness; Resistance Training; Training Intensity

\section{Introduction}

Based on training goals, for the variety of strength training methods there exist evidence-based and widely published recommendations to control training intensity (e.g.1, 8). However, widely unknown is the question, how far these recommendations for training intensity are implemented in fitness-related resistance training at commercial fitness clubs.Although about 10.61 million recreational athletes perform fitness-related resistance training in German fitness clubs [1], hardly any evidence can be found on strength training procedures respectively the control of training intensity of these fitness customers. The common used method to determine training intensity in fitness-related resistance training is to quantify the athletes' rate of perceived exertion - RPE [2]. In this approach, first the number of repetitions is determined based on the primary training goal. Subsequently, for this number of repetitions a training load is tested, which causes a previously defined rate of perceived exertion.

As justification for this approach it is argued that no muscle exhausting strength test (for example 1-RM-test) with accordingly high mechanical stresses for recreational athletes is necessary. Widely unexplained is the question, whether this training approach considers the scientifically substantiated recommendations for strength training intensity. There are some indications that RPEcontrolled training intensity leads to weight loads that are too low and therefor ineffective[3-5]. Due to thesefindings, it could be presumed that recreational athletes do not train with the required training intensity to reach their training targets. In order to acquire additional findings to this research field a multicenter-study over an investigation period of two years was deducted in 48 fitness clubs throughout Germany by the German University of Applied Sciences for Prevention and Health Management (DHfPG).

\section{Methods}

In a study of DHfPG to various research questions regarding training intensity in fitness-related strength training[2], it was examined inter alia, how far the RPE-based training intensities of recreational athletes correspond to the recognized scientific training recommendations.RPE-based strength training protocols 
using the Borg-Scale as well as strength training protocols with intuitive control of weight loads was object of this investigation. Experimental subjects were healthy adults performing fitnessand health-related resistance training. The total sample was stratified in various performance levels originating from strength training experience (from strength training beginners to advanced strength training practitioners). No representative minority groups (top athletes, children and adolescents younger than the age of 18) were not considered as experimental subjects. Exclusion criteria for participation included acute/chronic cardiovascular diseases, diseases of the respiratory system or diseases of the musculoskeletal system (tertiary prevention, rehabilitation).The primary recruitment aim was to acquire a representative clientele of commercial fitness clubs.

Training intensities of 211 recreational athletes (N=99 women, $\mathrm{N}=112$ men) were analyzed. All subjects voluntarily participated in the study and were able to withdraw from the investigation at any time. Concerning data privacy, all personal data were encoded. The research project was conducted according to the Declaration of Helsinki and approved by the internal Reviewing Board and Ethical Review Committee of DHfPG.Participants realized a six weeks standardized resistance training protocol with 2 or 3 training sessions per week (depending on performance level), 10 repetitions per set and a RPE-based training intensity. The following resistance training exercises were object of the investigation: horizontal leg press, chest press, butterfly, lat pulldown, horizontal row, dumbbell lateral raise, cable triceps pushdowns and dumbbell biceps curls. The primary research aim was to investigate strength gains caused by various RPE-based resistance training methods in fitnessrelated strength training, so inter alia 1-RM pre- and post-test were implemented. With the aid of 1-RM pre-test as comparative value, the average realized training intensity of the subjects was analyzed.

\section{Results}

Over a training period of six weeks, subjects trained with an average training intensity of $54.50 \%$ of 1 -RM $( \pm 6.58)$. The highest average training intensity was realized by horizontal leg press $(57.04 \% 1$-RM, \pm 5.79 ), thelowest by dumbbell biceps-curls (52.56\% 1-RM, 6.28).There was no significant difference in the average training intensity between samples using the Borg-Scale to control RPE and samples with intuitive control of weight load. Furthermore, there was no significant difference in the average training intensity between sexes.

Following the recognized recommendations, 10 repetitions should correspond from 75\% [6] to 80\% 1-RM [7].Overall, it can be stated that the analyzed recreational athletes trained with training intensities far off any scientific training recommendations. Notwithstanding this, significant strength gains (small to moderate effect sizes) for all examined RPE-based resistance training methods and all resistance exercises could be proved $[8,9]$.

\section{Discussion}

At this study recreational athletes trained with intensities, which do not comply with scientific training recommendations.
This could allow twofold conclusions. Firstly, it can be stated that recreational athletes trained with intensity levels far too low and therefore with ineffective training intensities. Secondly, the validity of widely published strength training recommendations regarding training intensity for the special population of recreational athletes in commercial fitness clubs has to be scrutinized. The fact that significant strength gains could be determined in spite of low intensities could be seen as an argument for the second thesis. At this point, it must be noted that recreational athletes in commercial fitness clubs pursue with their resistance training primarily preventive or aesthetic training targets. Such training targets result largely from structural adaptations of skeletal muscles. Strength gains quantified by RM-testing are no valid evidence for structural adaptations of skeletal muscles. Sport motorist strength gains are firstly functional effects and not necessarily the result of muscle hypertrophy processes. It must be clearly called into question if a training period of only six weeks, in combination with the realized low intensities can cause significant hypertrophy processes on skeletal muscles.Insofar, the thesis first mentioned can be assumed.

\section{Practical Applications}

From the results of the current study, it can be concluded that by using a RPE-based resistance training recreational athletes train with weight loads far too low. However, it would not be justified to constitute this strength training approach blanked as ineffective for fitness-related resistance training. Even with a more soft resistance training and low weight loads are the targeted training goals are gradually attainable. Though the available studies (e.g. 7) show clearly, that resistance training methods with intensities corresponding to recognized training scientific recommendations result in significantly higher training effects. Even in fitnessrelated resistance training should be considered more strongly to implement strength training methods following a RM-based control of training intensity to reach the training goals of the fitness customers in a more effective and efficient way. In this respect, it could be derived an optimization potential for fitness-related resistance training in commercial fitness clubs from the current study results.

\section{Acknowledgement}

The author would like to thank all subjects for their outstanding and dedicated collaboration. The author has no professional relationship with companies or manufactures that might benefit from the results of this study. There was no financial support for this project. Therefore, the author declares no conflict of interest.

\section{References}

1. (2018) German Sports Club Association (Deutscher Sportstudio Verband) Benchmark data of the German fitness industry 2018 (Eckdaten der deutschen Fitnesswirtschaft 2018). Hamburg, Germany.

2. Eifler C (2017) Control of intensity in fitness-related resistance trainingan empirical study (Intensitätssteuerung im fitness or ientierten Krafttraining-eine empirische Studie). Marburg, Germany.

3. Focht BC (2007) Perceived exertion and training load during selfselected and imposed-intensity resistance exercise in untrained women. J Strength Cond Res 21(1): 183-187. 
4. Glass S (2008) Effect of a learning trial on self-selected resistance training intensity. J Strength Cond Res 22(3): 1025-1029.

5. Ratamess NA, Faigenbaum AD, Hoffman JR, Kang J (2008) Self-selected resistance training intensity in healthy women: The influence of a personal trainer. J Strength Cond Res 22(1): 103-111.

6. Baechle TR, Earle RW, Wathen D (2008) Resistance training. In Baechle TR, Earle RW (Eds.). Essentials of strength training and conditioning $3^{\text {rd }}$ (Edn.). Human Kinetics, Champain, IL, US, pp. 381-412.
7. Eifler C (2016) Short-term effects of different loading schemes in fitnessrelated resistance training. J Strength Cond Res 30(7): 1880-1889.

8. Peterson MD, Rhea MR, Alvar BA (2004) Maximizing strength development in athletes: A meta-analysis to determine the doseresponse relationship. J Strength Cond Res 18(2): 377-382.

9. Ratamess NA, Alvar BA, Evetoch TK, Housh TJ, Kibler WB, et al. (2009) American College of Sports Medicine position stand. Progression models in resistance training for healthy adults. Med Sci Sports Exerc 41(3): 687-708.

Assets of Publishing with us
BIOMEDICAL $\quad \begin{aligned} & \text { Global archiving of articles } \\ & \text { RESEARCHES }\end{aligned}$

